\title{
5 Die Bibliothek Philodems: Inhalt und Bedeutung für die Klassische Philologie
}

Est quidam Graecus, qui cum isto vivit, homo, vere ut dicam - sic enim cognovi - humanus ... Est autem hic de quo loquor non philosophia solum sed etiam ceteris studiis, quae fere ceteros Epicureos neglegere dicunt, perpolitus...

Cicero über Philodem, In Pisonem (55 v. Chr.)

\subsection{Hoffnung auf verlorene Meisterwerke und Philodem}

Waren unter den Papyri verlorene Meisterwerke der Antike? Winckelmann schrieb 1762, als erst vier Papyri, darunter ein Buch von Philodems De musica, entrollt waren: „Man wünschte Geschichtsschreiber zu finden, wie die verlorenen Bücher des Diodorus, die Geschichte des Theopompus und des Ephorus und andere Schriften, als des Aristoteles Beurteilung der dramatischen Dichter, die verlorenen Tragödien des Sophokles und des Euripides, die Komödien des Menanders und des Alexis, die Symmetrie des Pamphilus für die Maler, und einige Werke von der Baukunst: an einer hypochondrischen und zerstümmelten Klage wider die Musik ist uns nicht viel gelegen.“" ${ }^{47}$ Die Wunschlisten anderer Zeitgenossen variierten je nach persönlichem Gusto. ${ }^{48}$ Epikureische Schriften standen auf niemandes Wunschliste. Jedoch stellte sich in den folgenden Jahrzehnten heraus, dass es sich bei der griechischen Bibliothek der Villa um die des Epikureers Philodem von Gadara handelte, welcher die von Winckelmann geschmähte Schrift verfasste. Dutzende Rollen weisen Philodem als Autor aus und auch Entwurfsfassungen seiner Bücher wurden gefunden. Wie es sich für einen Epikureer gehört, enthielt seine Bibliothek allen voran Bücher Epikurs und berühmter Epikureer, welche seit der Spätantike nicht mehr überliefert wurden.

Philodem (etwa 110-30 v. Chr.) ${ }^{49}$ dürfte seine hellenistisch geprägte Heimatstadt Gadara in der Dekapolis (heutiges Jordanien) um 90 v. Chr. verlassen

47 Winckelmann, Sendschreiben (wie Anm. 24), 89-90.

$48 \mathrm{Vgl}$. Sider, The Library (wie Anm. 5), 63; Longo Auricchio et al., La villa (wie Anm. 5), 70.

49 Für Philodems Biographie siehe Enzo Puglia, „Perché Filodemo non fu ad Alessandria?,“ SEP 1 (2004): (133-138); Kilian Fleischer, „Dating Philodemus’ birth and early studies,“ BASP 55 (2018): (119-127); Francesca Longo Auricchio, „Qualche considerazione sulla biografia di Filodemo,“ CErc 49 (2019): (31-38); Blank, Philodemus (wie Anm. 5), Kapitel: „life“; David Armstrong und Michael McOsker, Philodemus. On Anger (Atlanta, 2020), 1-10. 
haben und weilte zunächst einige Zeit in Alexandria. Um 85 v. Chr. siedelte er nach Athen über, um dort bei dem Leiter (Scholarch) der epikureischen Schule Zenon von Sidon (160-75) ${ }^{50}$ im кñлos (Garten) philosophischen Studien nachzugehen. Nach Zenons Tod verließ Philodem etwa zwischen 75 und 70 v. Chr. Athen und ging nach Italien, vielleicht anfangs nach Sizilien. $\mathrm{Zu}$ dieser Zeit, womöglich aber schon vorher, muss er mit seinem späteren Patron Lucius Calpurnius Piso Caesoninus, Konsul des Jahres 58 v. Chr., in Kontakt gekommen sein. Vermutlich begleitete Philodem Piso auf einigen Reisen und lebte sowohl in Rom als auch am Golf von Neapel in dessen Umfeld. Piso ist in einem von Philodems Epigrammen adressiert ${ }^{51}$ und auch das Werk De bono rege secundum Homerum ist dem Politiker gewidmet. Cicero rekurriert auf Philodem als Pisos „Hausphilosophen“ in seiner Rede gegen den vormaligen Konsul (In Pisonem). Er charakterisiert Philodem bei aller Polemik doch als kultivierten Epikureer. ${ }^{52}$ Auch eine Stelle in De finibus spricht für Ciceros Wertschätzung des Philosophen. ${ }^{53}$ Horaz erwähnt Philodem ebenfalls. ${ }^{54}$ Philodem war es, der mit anderen die epikureische Philosophie in Rom und Italien einbürgerte. ${ }^{55}$ Er stand mit Siro sowie den „Augusteern“, Vergil, Plotius Tucca, Quinctilius Varo und Varius Rufus in freundschaftlichem Kontakt - alle fünf sind in Herkulanischen Papyri erwähnt. ${ }^{56}$ Philodem hat auch Einfluss auf deren Dichtung und epikurische Prägung ausgeübt. ${ }^{57}$ Über eine direkte Beziehung Philodems zu Horaz und Lukrez wird diskutiert. Philodem legte größeren Wert auf Ethik als auf Naturphilosophie, was eine Konzession an sein eher praktisch orientiertes römisches Umfeld gewesen sein dürfte. Seine schriftstellerischen Qualitäten werden heute zusehend positiver bewertet, aber „schön zu lesen“ ist das Griechisch meistens

50 Für seine Lebensdaten siehe Kilian Fleischer, „Zenone di Sidone nacque intorno al 160 a.C.,“ RFIC 147 (2019): (43-50).

51 Philodem, Epigramm 27 Sider (=AP 11,44).

52 Cicero, In Pisonem 68-72,74 (hg. von Robin Nisbet, M. Tulli Ciceronis in L. Calpurnium Pisonem oratio. Edited with Text, Introduction, and Commentary [Oxford 1961] =T 2 Sider).

53 Cicero, De finibus 2,119 (... familiares vestros, credo, Sironem dicis et Philodemum, cum optimos viros tum homines doctissimos) (OCT, 95,1-3 Reynolds =T 1 Sider).

54 Horaz, Sermones 1,2,119-122 (OCT, 141 Garrod =T 4 Sider).

55 Vgl. David Sedley, „Philodemus and the decentralisation of philosophy,“ CErc 33 (2003): (31-41).

56 Siro in PHerc. 312, col. 14 (=T 15 Sider), die genannten Augusteer in PHerc. Paris. 2, PHerc. 253 und PHerc. 1082. $\mathrm{Zu}$ Philodem und den Augusteern vgl. David Armstrong/Jeffrey Fish/Patricia Jonston/Marilyn Skinner (Hgg.), Vergil, Philodemus, and the Augustans (Austin, 2004).

57 Vgl. Richard Janko, Philodemus. On Poems, Book 2. With the fragments of Heracleodorus and Pausimachus (Oxford, 2020), 162-166. 
nicht. Bis zur Entdeckung der Herkulanischen Papyri war Philodem als Autor nur durch rund 35 Epigramme in der Anthologia Palatina bekannt.

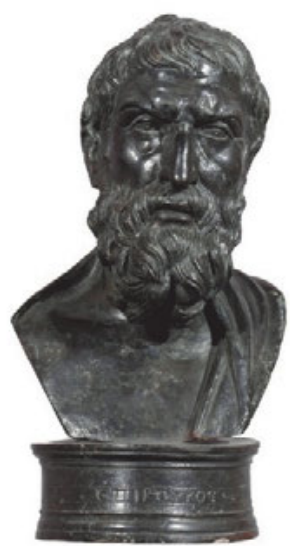

Abb. 16: Büste Epikurs (im Tablinum der Villa gefunden)

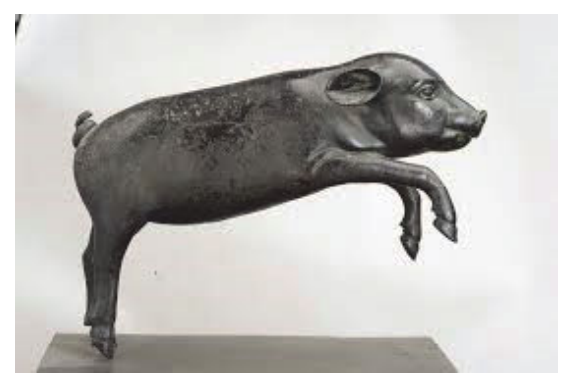

Abb. 17: Das Schweinchen hat wohl einen Bezug zum Epikureismus (im großen Peristyl der Villa gefunden)

\subsection{Inhalt der Herkulanischen Bibliothek}

In der „Bibliothek“ der Villa - größtenteils die Bibliothek Philodems - wurden insbesondere folgende Autoren und Werke identifiziert: ${ }^{58}$

58 Für eine andere Übersicht zu den gefundenen Werken siehe Agnese Travaglione, Catalogo descrittivo dei papiri Ercolanesi (Neapel, 2008), 320-328; Blank, Philodemus (wie Anm. 5); Longo Auricchio et al., La villa (wie Anm. 5), 137-179. Die lateinischen Bezeichnungen gehen im Wesentlichen auf Erler, Epikur (wie Anm. 5) zurück und variieren bisweilen in Publikationen (teils sind die originalen Titel nicht erhalten und nur „Pseudo-Titel“ aufgrund des Inhalts zugewiesen). Zu den Titeln (subscriptiones) der Werke siehe Gianluca Del Mastro, Titoli e annotazioni bibliologiche nei papiri greci di Ercolano (Neapel, 2014). Aktualisierte griechische Titel und gängige Abkürzungen finden sich am Ende aller CErc-Bände sowie auf www.chartes.it (siehe 13.2.2). Die Buchzuweisung und auch Werkzuweisung einzelner Papyri wird diskutiert. Auch die Kategorisierung bzw. Einteilung von Philodems Schriften wird verschiedentlich vorgenommen. Ich habe in meiner Aufstellung frühere Ansätze synthetisiert. 


\section{Epikureer}

\section{Epikur}

De natura: Buch 2 (PHerc. 1149/993+1783/1691/1010), 9 (PHerc. 560), 10 (PHerc. 1413/1416), 11 (PHerc. 1042+154), 14 (PHerc. 1148), 15 (PHerc. 1151), 21 (PHerc. 362), 25 (PHerc. 454/1420/1056+697+1191), 28 (PHerc. 1479/1417), 34 (PHerc. 1431) Teils substantielle Reste nicht genauer bestimmbarer Bücher des Werkes finden sich unter diversen anderen PHerc.-Nummern.

Echelaos (PHerc. 566)

\section{Metrodor}

De divitïs (PHerc. 200)

Contra dialecticos (sophistas) (PHerc. 255,418,439,456,1084,1091,1108,1112,1645, $1788)^{59}$

\section{Polyainos}

Contra Aristonem (PHerc. 573)

\section{Kolotes}

Contra Platonis Lysin (PHerc. 208)

Contra Platonis Euthydemum (PHerc. 1032)

\section{Polystratos}

De iniusto contemptu (PHerc. 336/1150)

De philosophia (PHerc. 1520)

\section{Karneiskos}

Philistas (PHerc. 1027)

\section{Zenon von Sidon}

Contra Craterum de geometria (PHerc. 1533)

\section{Demetrius Laco}

Quaestiones convivales (PHerc. 1006)

Ad Polyaeni dubia (PHerc. 1083,1258,1429,1642,1647,1822)

De geometria (PHerc. 1061)

59 Für die Nummern vgl. Kilian Fleischer, „Die ältesten Papyri Herkulaneums: PHerc. 1788 und andere Metrodorpapyri,“ CErc 49 (2019): (17-29). 
De poematis (PHerc. 188, 1014)

De sole (PHerc. 1013, PHerc. 177) ${ }^{60}$

De elatione animi (PHerc. 831)

Quaestiones exegeticae (PHerc. 1012)

De forma dei (PHerc. 1055)

\section{Philodem}

a) Philosophiehistorische Schriften

Syntaxis philosophorum

Index Academicorum (PHerc. 1021/1691 + 164)

Index Stoicorum (PHerc. 1018)

Index Epicureorum (PHerc. 1780)

Vita Socratis (PHerc. 495+558)

Index Eleaticorum et Abderitum (PHerc. 327- unsicher)

Index Pythagoreorum (PHerc. 1508- unsicher)

De Stoicis (PHerc. 155+339)

b) Historiographie zu Epikur/Epikureismus

De Epicuro (PHerc. 1232,1289)

De mentionibus in Epicuro / Pragmateiai (PHerc. 1418+310) ${ }^{61}$

Vita Philonidis (PHerc. 1044/1746/1715)

\section{c) Ästhetische Schriften}

De rhetorica (Dutzende PHerc.-Nummern - am besten erhaltene Werk Philodems, mehrere Bücher)

De musica (PHerc. 1497 und etliche andere PHerc.-Nummern, mehrere Bücher)

De poematis (Dutzende PHerc.-Nummern - 5 Bücher - viel erhalten) ${ }^{62}$

$60 \mathrm{Zu}$ PHerc. 177 siehe Graziano Ranocchia, „Un nuovo titolo iniziale nella collezione ercolanese e un nuovo libro (del trattato) sul Sole di Demetrio Lacone (P.Herc. 177),“ Aegyptus 98 (2018): (3-36).

61 Zum Titel des Werkes, welcher kaum den Plural $\pi \rho \alpha \gamma \mu \alpha \tau \varepsilon i ̃ \alpha$ enthielt, siehe Enzo Puglia, „Le cosiddette Pragmateiai di Filodemo, ovvero dell' immeritata fortuna di un titolo,“ in è sì d“ amici pieno. Ommagio di studiosi italiani a Guido Bastianini per il suo settantesimo compleanno (hg. von Angelo Casanova/Gabriella Messeri/Rosario Pintaudi; Florenz, 2016), (309-315) und Kilian Fleischer, „Epikur, Philodem, ПРАГМАТЕIAI: Ein neuer Akzent in Titel und Bewertung von PHerc. 1418+310,“ ZPE 210 (2019): (54-70).

$62 \mathrm{Zu}$ den Nummern siehe Janko, Philodemus‘ On Poetry (wie Anm. 57), 6-7. 
d) Ethische Schriften

De vitiis

De adulatione (PHerc. 222,223/1082/1089/1675,1457)

De calumnia (PHerc. Paris.2)

De oeconomia (PHerc. 1424)

De superbia (PHerc. 1008)

De divitiis (PHerc. 163)

De gratia (PHerc. 1414)

De homilia (PHerc. 873/1399)

De ira (PHerc. 182)

De morte (PHerc. 1050)

Adversus eos qui se libros nosse profitentur (PHerc. 1005/862+1485)

De bono rege secundum Homerum (PHerc. 1507)

De electionibus et fugis (PHerc. 1251)

De sensibus (PHerc. 19/698)

De vitis

De libertate dicendi (PHerc. 1471)

e) Theologische Schriften

De dis (PHerc. 26, 152/157)

De pietate (PHerc. 1428)

De providentia (PHerc. 1670/1100/1577/1579) ${ }^{63}$

f) Epistemologische Schriften

De signis (PHerc. 1065)

Ferner sind zahlreiche als „opus incertum“ bezeichnete Papyri Philodem zuzuweisen. Teils ist auch der Inhalt näher eingrenzbar.

\section{Stoiker}

\section{Chrysipp}

Quaestiones logicae (PHerc. 307)

De providentia (PHerc. 1038,1421)

63 Vgl. Claudio Vergara, „I papiri dell‘ opera De Providentia di Filodemo,“ CErc 50 (2020): (9199). 
Einige andere Rollen stoischen Inhalts sind wahrscheinlich auch chrysippeisch (PHerc. 1020, $\left.{ }^{64} 1384,1158\right)$.

\section{Lateinische Papyri}

Anonym, De Bello Actiaco (PHerc. 817)

Seneca d. Ältere, Historiae ab initio bellorum civilium (PHerc. 1067)

Die meisten anderen lateinischen Papyri sind nur sehr fragmentarisch erhalten und oftmals kann nicht einmal eine wahrscheinliche These zum Inhalt aufgestellt werden.

Bei einigen der aufgezählten Schriften wurden „kleinere“ oder „unsichere“ Fragmente (PHerc.-Nummern) nicht angegeben, so dass sich die einzelnen Nummern meist auf den relativ gut erhaltenen Mittelteil der Rollen (midollo) beziehen. Die meisten der gelisteten Werke bestehen aus mehreren Büchern, was nicht angegeben wurde, da die Gesamtzahl der Bücher bei vielen Werken unbekannt ist und eine genauere Aufschlüsselung der Nummern hier zu unübersichtlich geworden wäre, zumal viele Zuordnungen und Buchzahlen in der Forschung diskutiert werden (bspw. bestand De morte aus mindestens 4 Büchern, De vitiis aus mindestens zehn Büchern, usw.). Auch nicht angeführt sind etliche (hunderte) der insgesamt 1840 PHerc.-Nummern (und einige Sonderinventarisierungen), unter denen nur sehr fragmentarische Reste aufbewahrt sind, so dass eine Zuweisung an einen Autor oder ein Werk oder auch nur eine wahrscheinliche Aussage zum Inhalt nicht möglich ist. Im Falle von Epikurs De natura sieht man exemplarisch, dass sehr oft mehrere PHerc.-Nummern einem Werk zugeordnet sind, da Teile von ein und derselben Rolle im Zuge der Arbeiten (Zerbrechen der Rolle in mehrere Stücke) und Archivierung in der frühen Neuzeit „chaotisch“ auf mehrere Nummern (cornici) verteilt wurden (siehe 13.1.2). Das Pluszeichen (+) bedeutet, dass von ein und demselben Werk mehrere Kopien (Versionen) in der Bibliothek vorhanden waren, während der Gedankenstrich (-) anzeigt, dass die Bücher des übergeordneten Werkes diese (fiktiven) Titel tragen.

Bei einigen philodemeischen Werken ist es aufgrund des Erhaltungszustands nicht ausgeschlossen, dass es sich wie im Falle des Index Academicorum

64 Michelle Alessandrelli und Graziano Ranocchia, Scrittore stoico anonimo, Opera incerta (PHerc. 1020), coll.104-112. Edizione, introduzione e commento (Rom, 2017). 
(siehe 15.1) um vorläufige Arbeitsfassungen handelt. Epikurs 37 Bücher umfassendes Monumentalwerk $\pi \varepsilon \rho i ~ \varphi v ́ \sigma \varepsilon \omega \varsigma$ befand sich wohl in Komplettfassung in der Herkulanischen Bibliothek; die Reste erlauben uns nun auch einen erweiterten Vergleich mit Lukrezens Disposition des Stoffes in De rerum natura. Von weiteren, hier nicht aufgeführten Werken Philodems kennen wir aus Zitaten in eigenen Schriften den Titel und auch für andere, hier nicht aufgeführte kleinere, d.h. sehr schlecht erhaltene Papyri (Philodems) wurden mitunter provisorische Titel konjiziert. Einige Papyri mit Werken Epikurs und Metrodors repräsentieren sehr alte Kopien und wurden lange vor Philodem im 3. Jh. v. Chr. niedergeschrieben. ${ }^{65}$ Im Falle von Epikurs De natura könnten Mehrfach-Kopien desselben Buches mit philologisch-überlieferungsgeschichtlichen Interessen und Studien Philodems zusammenhängen, der durch Vergleich den korrekten Urtext sichern wollte. Offenbar nahm Philodem einige Papyri aus Athen, wohl aus dem Umfeld der dortigen Schule, mit nach Italien.

Die Länge der erhaltenen Papyri bzw. ihre erhaltene Kolumnenzahl variiert erheblich zwischen den einzelnen PHerc-Nummern. Von manchen Werken sind lediglich wenige Zeilen deutlich lesbar oder fast nur der Titel und Verfassername am Ende der Rolle - die subscriptio - erhalten, ${ }^{66}$ während unter anderen Nummern zahlreiche Kolumnen in der Größenordnung dutzender moderner Norm-Seiten zu finden sind.

Die lateinischen (entrollten) Papyri summieren sich auf weniger als 100 (Rollen) und datieren wohl überwiegend aus der Zeit nach Philodem. Sie sind fast ausnahmslos sehr schlecht erhalten, was vermuten lässt, dass sie während des Ausbruchs größtenteils nicht in unmittelbarer Nähe zu den besser erhaltenen griechischen Papyri verwahrt waren. ${ }^{67}$ Entgegen den Behauptungen von Kleve und anderen in den 1990er Jahren (und darüber hinaus) sind keine Reste von Lukrezens De rerum natura mit Sicherheit unter den lateinischen Papyri auszumachen. Ebenso steht die vermutete Präsenz von Ennius und Caecilius Statius zur Diskussion. ${ }^{68}$ Die jüngste Wiederherstellung der subscriptio von

65 Vgl. Guglielmo Cavallo, Libri scritture scribe a Ercolano (Suppl. CErc 13) (Neapel, 1982), 5758.

$66 \mathrm{Zu}$ den Titeln der Herkulanischen Werke bzw. den subscriptiones und stichometrischen Fragen siehe grundlegend Del Mastro, Titoli (wie Anm. 58).

67 Vgl. Capasso, Custodia e lettura (wie Anm. 34), 14.

68 Guglielmo Cavallo, „I papiri di Ercolano come documenti per la storia delle biblioteche e dei libri antichi,“ Atti dell Accademia nazionale dei Lincei, serie IX, XXXV, 3, Rom (2015): (573598), 594 und Mario Capasso, Les papyrus latins d' Herculanum. Découverte, consistence, contenu (Liege, 2011), 63 gegen die Ausführungen von Knut Kleve, „Lucretius in Herculaneum,“ CErc 19 (1989): (5-27) und Knut Kleve, „Ennius in Herculaneum,“ CErc 20 (1990): (5-16). 
PHerc. 1067 weist den Papyrus als Werk Senecas des Älteren aus, was zeigt, dass zumindest der lateinische Teil der Bibliothek (fast) bis zum Vesuvausbruch „up-to-date“ gehalten wurde. ${ }^{69}$

\subsection{Wert und Bedeutung der Papyri}

Die primäre Bedeutung der Papyri liegt darin, dass sie uns einen vertieften, authentischen Einblick in die epikureische Philosophie und ihre literarischen Spielarten gewähren, also in eine Philosophierichtung, deren Schriften das spätantike Nadelöhr der Überlieferung nicht passiert haben und fast gänzlich verloren gingen. Allerdings relativiert bzw. verbreitert sich der anhand obiger Autorenliste dominierende epikureisch-spezielle Charakter der Bibliothek, wenn man bedenkt, dass Philodem die Ansichten anderer Philosophen vor deren Widerlegung oft sehr ausführlich paraphrasiert oder zitiert. Die Herkulanischen Papyri sind eine wahre Fundgrube für viele anderweitig verlorene nicht-epikureische Autoren, unter ihnen Philosophen, Historiker, Grammatiker und Dichter. Wir sprechen hier von einer solchen Quantität, dass etwa gegenwärtig ein voluminöses Corpus Praesocraticorum Herculanensium im Erscheinen begriffen ist, welches beanspruchen kann, eine gewichtige Ergänzung zu den Vorsokratikerfragmenten von Diels-Kranz zu werden. ${ }^{70}$ Ferner profitier(t)en etwa auch Jacobys Fragmente der Griechischen Historiker und viele andere „Corpora“ in erheblichem Maße von den Papyri. Selbst die athenische Archontenliste erfuhr durch die Papyri manche Erweiterung, was die Strahlkraft der Herkulanischen Papyri auf andere Gebiete der Altertumswissenschaften versinnbildlicht. ${ }^{71}$ Trotz der unbestreitbar epikureischen Präponderanz wäre es gänzlich verfehlt, die „Herkulanischen Papyri“ auf die Bezeichnung „Epikureische Papyri“ zu reduzieren und damit eine thematische oder literarische Enge $\mathrm{zu}$ insinuieren, welche die Papyri ob ihrer nicht marginalen Bedeutung für For-

69 Vgl. Longo Auricchio et al., La villa (wie Anm. 5), 137-138. Viele lateinische Papyri wurden im Zuge des ERC-Projekts PLATINUM (Papyri and LAtin Texts: INsights and Updated Methodologies Towards a philological, literary, and historical approach to Latin papyri) näher untersucht. Siehe die Datenbank „Il nuovo Corpus of Latin Texts on Papyrus (CLTP)“. Zu dem Werk Senecas des Älteren siehe Valeria Piano, „Il P. Herc. 1067 latino: il rotolo, il testo, l'autore,“ CErc 47 (2017): (163-250) und Maria Scappaticcio (Hg.), Seneca the Elder and his Rediscovered „Historiae ab initio bellorum civilium“ (Berlin, 2020).

70 Christian Vassallo, The Presocratics at Herculaneum: A Study of Early Greek Philosophy in the Epicurean Tradition (= Studia Praesocratica, 11) (Berlin, 2021).

71 Tiziano Dorandi, „Gli arconti nei papiri ercolanesi,“ ZPE 84 (1990): (121-138). 
schungszweige jenseits des Epikureismus keineswegs haben. Beinahe unnötig zu erwähnen ist, dass die Rollen für bibliologische (mise en page, Stichometrie), buchgeschichtliche und paläographische Studien von besonderem Aussagegehalt sind und die Befunde aus Ägypten einordnen und ergänzen. Folglich sollten wir nicht in die Klagen mancher Gelehrter des 19. Jahrhunderts über das (vorläufige) Ausbleiben erhoffter Meisterwerke einstimmen und uns für das unverhofft Erhaltene glücklich schätzen. ${ }^{72}$

72 Siehe etwa Domenico Comparetti und Giulio De Petra, La Villa ercolanese dei Pisoni: I suoi monumenti e la sua biblioteca (Neapel, 1883), 79; Charles Waldstein und Leonhard Shoobridge, Herculaneum: Past, Present, Future (London, 1908), 83. 